\title{
1 Landscape genetics and species delimitation in the Andean palm rocket frog,
}

\section{Rheobates spp.}

4 Gabrielle Genty1*, Carlos E. Guarnizo1, Juan P. Ramírez1,2, Lucas Barrientos1,3, Andrew J.

5 Crawford 1

6 1Department of Biological Sciences, Universidad de los Andes, Bogotá, 111711, Colombia.

72 Department of Biology, San Diego State University, San Diego, CA, 92182, USA.

83 Universidad del Rosario, Departamento de Biología, Bogotá, 111221, Colombia.

$9 *$ Communicating author: Gabrielle Genty

10 Departamento de Ciencias Biológicas,

11 Universidad de los Andes

12 Calle 19 No. 1-60

13 Bogotá, código postal 111711, Colombia, South America

14 Email: gy.genty180@uniandes.edu.co

15 Alt. email: gabygenty@gmail.com

16 


\section{Abstract}

19 The complex topography of the species-rich northern Andes creates heterogeneous

20 environmental landscapes that are hypothesized to have promoted population fragmentation and

21 diversification by vicariance, gradients and/or the adaptation of species. Previous phylogenetic

22 work on the Palm Rocket Frog (Anura: Aromobatidae: Rheobates spp.), endemic to mid-

23 elevation forests of Colombia, suggested valleys were important in promoting divergence

24 between lineages. In this study, we use a spatially, multi-locus population genetic approach of

25 two mitochondrial and four nuclear genes from 25 samples representing the complete geographic

26 range of the genus to delimit species and test for landscape effects on genetic divergence within

27 Rheobates. We tested three landscape genetic models: isolation by distance, isolation by

28 resistance, and isolation by environment. Bayesian species delimitation (BPP) and a Poisson

29 Tree Process (PTP) model both recovered five highly divergent genetic lineages within

30 Rheobates, rather than the three inferred in a previous study. We found that an isolation by

31 environment provided the only variable significantly correlated with genetic distances for both

32 mitochondrial and nuclear genes, suggesting that local adaptation may have a role driving the

33 genetic divergence within this genus of frogs. Thus, genetic divergence in Rheobates may be

34 driven by the local environments where these frogs live, even more so that by the environmental

35 characteristics of the intervening regions among populations (i.e., geographic barriers).

\section{Keywords}

38 Colombia, genetic distance, isolation by distance, isolation by environment, landscape genetics, 39 phylogeography. 


\section{Introduction}

The South American landscape and environments have experienced enormous historical changes, such as major geological upheavals, environmental fluctuations and glaciations (Graham, 2009), which have created a complex backdrop for species diversification. The tropical Andes are a global biodiversity hotspot, home to the highest density of species per unit area in the world (Hutter et al., 2017; Morueta-Holme et al., 2015; Myers et al., 2000). In contrast to the high alpha-diversity in Amazonia, the Andes exhibit high beta-diversity, attributed to the topographic and climatic heterogeneity resulting from Andean orogenesis (Jaramillo et al., 2006). The Andean Cordillera has a dynamic history, progressively uplifting from South to North during the Late Cretaceous (Folguera \& Ramos, 2011; Hoorn et al., 2010; Horton et al., 2010; Leier et al., 2013; Mescua et al., 2013). This orogenic uplift and its concomitant environmental heterogeneity have promoted the diversification of Andean species (Graham, 2009; Hoorn et al., 2010).

One way in which the complex topography of the Andes promote species isolation and diversification is by fragmenting lowland populations or by limiting dispersal across high elevation regions split by valleys or mountain peaks (Ghalambor et al., 2006). For example, the Andean valleys promoted the diversification of the montane forest subspecies of three-striped warbler bird, suggested to occur by allopatric divergence (Gutiérrez-Pinto et al., 2012), and the Adelomyia hummingbirds, whose divergence was coupled to Andean orogeny (Chaves et al., 2011). Moreover, Andean peaks promoted lowland speciation in general, such as the Dendrocincla woodcreepers, through vicariance during the uplift (Weir \& Price, 2011). Another way the Andes can promote diversification is by providing novel environments at different elevations, which in turn could promote local adaptation and divergence (Schluter, 
63 2000). Previous studies on birds supported that environmental gradients along elevation belts

64 have promoted trait evolution (P. R. Grant \& Grant, 2011; Luzuriaga-Aveiga \& Weir, 2019).

65 Hence, elevation can promote differentiation among populations but whether this differentiation

66 is leading to speciation is controversial.

Even though some studies suggest that the Andean topography promotes genetic

divergence and diversification in the tropics (Chaves et al., 2011; Gutiérrez-Pinto et al., 2012;

69 Weir \& Price, 2011), further investigation is needed to understand the origins of this diversity. In

70 a recent pre-print, Rodríguez-Muñoz et al.(2020) suggested that environment, rather than Andean

71 uplift is the main factor explaining genetic divergence across lowland tetrapod species separated

72 by the Eastern Andes. However, when invoking environment as a barrier, there are two factors to

73 consider: one is the more typical assumption that the environment of the intervening region is

74 what isolates pairs of populations, while the second possibility is the distinctiveness of the

75 environment that characterizes the respective populations. The latter scenario is referred to as

76 isolation by environment and suggests that populations inhabiting contrasting environments have

77 limited dispersal due to either selection against immigrants or due to individuals preferring to

78 remain in a particular environment (Wang et al., 2013), as has been found to occur in the

79 European Common Frog (Rana temporaria) in Scotland (Muir et al., 2014). In other words, the

80 isolation by environment model is blind to the environmental landscape conditions found in

81 between a pair of populations, and therefore contrasts sharply with a more traditional geographic

82 barrier scenario or an isolation by resistance model (McRae, 2006).

83 Tropical amphibians are ideal organisms to evaluate how geography and environment are

84 associated with diversification in montane regions due to their restricted dispersal, strong site

85 fidelity, and spatially isolated breeding habitat (Beebee, 2005; Feder \& Burggren, 1992; Smith \& 
Green, 2005). The Palm Rocket Frog (Anura: Aromobatidae: Rheobates) is a useful system to

87 investigate how Andean topography has promoted biological diversification. The genus

Rheobates is endemic to Colombia, found only in the Eastern and Central Andean cordilleras of

Colombia, and restricted to mid-elevation habitats between 250 and $2520 \mathrm{~m}$ above sea level

(Bernal \& Lynch, 2008; Ovalle-Pacheco et al., 2019). Rheobates is currently composed of two

91 species, $R$. palmatus (Werner, 1899) found in the Eastern and Central Cordilleras of Colombia,

92 and R. pseudopalmatus (Rivero \& Serna, 1995) found only in the northern part of the Central

93 Cordillera of Colombia. However, the taxonomic status of the latter taxon merits further scrutiny

94 (Grant et al. 2006, 2017) due to the apparent absence of adequate morphological characters

distinguishing it from $R$. palmatus. Furthermore, recent molecular phylogenetic studies (Grant et

98 pseudopalmatus than to R. palmatus samples from the Eastern Cordillera.

99 In a previous phylogenetic study, using two mitochondrial genes and one nuclear gene,

100 Muñoz-Ortiz et al. (2015) found that Rheobates was composed of three highly divergent clades.

101 The lineage located in Santa María, Boyacá, on the eastern side of the Eastern Cordillera was

102 isolated from the rest of the genus during the early Miocene, and the remaining populations

103 formed two reciprocally monophyletic groups separated by the Magdalena River Valley.

104 However, the authors did not employ statistically robust, multi-locus species delimitation

105 methods to evaluate the hypothesis of Rheobates being comprised of three lineages. Moreover,

106 phylogenies with mitochondrial and nuclear DNA can present discordances (Edwards \& Bensch,

107 2009; Toews \& Brelsford, 2012). Therefore, analyses which utilize the genealogical

108 independence of multiple loci are needed to confirm phylogeographic histories. For this reason, 
109 in the present study we used three new nuclear markers and complemented the previously

110 amplified mitochondrial and nuclear DNA data for some samples (Table S1). Muñoz-Ortiz et al.

111 (2015) also suggested that the genetic divergence within Rheobates is caused by geographic

112 barriers with environmental properties that lay outside the niche of this genus, such as the aridity

113 in the Magdalena Valley or the low temperatures characteristic of the elevations above 2000

114 meters, yet the authors offered no spatial genetic analyses nor explicit tests for the role of

115 environment versus geography in promoting divergence within the genus.

116 In this study, we use a) multilocus, coalescent-based species delimitation approaches and

117 model-based historical demographic inference to evaluate the phylogeographic structure within

118 the genus Rheobates, and b) a landscape genetics approach to investigate the role of physical

119 geography and environmental variation as potential drivers of genetic differentiation among

120 populations of Rheobates. Specifically, we tested whether the pattern of divergence in Rheobates

121 across the landscape fits a model of isolation by distance (Wright, 1943), isolation by resistance

122 (Wang et al., 2009), or isolation by environment (Wang et al., 2013; Wang \& Bradburd, 2014).

123 Support for a pattern of isolation by distance would indicate that geographic distance among

124 populations explains genetic divergence within Rheobates (Wright, 1943). Support for a pattern

125 of isolation by resistance would indicate that connectivity among populations is affected by

126 environmental barriers perhaps related to the heterogenous Andean topography, i.e., areas with

127 abiotic characteristics less suitable for Rheobates (McRae, 2006). Support for isolation by

128 environment, however, would indicate that individuals would be more closely related (lower

129 genetic distance) the more similar their environments are, independently of the geographic or

130 'resistance' distances between populations, which may indicate local adaptation (Wang \&

131 Bradburd, 2014). 
133 and expanded the genetic sampling from one to four nuclear markers. We also included new

134 samples from a highly divergent locality form the eastern side of the Eastern Cordillera, detected

135 by Muñoz-Ortiz et al. (2015).

137 Methods

\section{Molecular genetic analyses}

$140 \quad$ Genomic DNA from 24 tissue samples of Rheobates were obtained through museum

141 donations from the tissue collection ANDES-T of the Museo de Historia Natural C. J.

142 Marinkelle at the Universidad de los Andes (see Table S1 in Supplementary Material) by using a

143 DNeasy Blood \& Tissue kit (Qiagen, Valencia, CA, USA) following the manufacturer's

144 protocol. For phylogenetic analysis, we included as outgroups, two samples from the aromobatid 145 genus, Allobates, thought to be closely related to Rheobates (T. Grant et al., 2006; Santos et al.,

146 2009). We used the previously published sequences (Muñoz-Ortíz et al., 2015) of two

147 mitochondrial genes, cytochrome oxidase I (aka, the COI Barcode of Life; Hebert et al., 2003)

148 for 23 samples and 16S ribosomal RNA (16S) for 22 samples. We included new samples which

149 were successfully amplified by polymerase chain reaction (PCR), making in total 24 samples for

150 COI and 25 samples for 16S (Table S2). Additionally, we included the previously published

151 sequences (Muñoz-Ortíz et al., 2015) of 18 samples for the nuclear gene proopiomelanocortin

152 (POMC) and sequenced new samples, making in total 22 samples for POMC. We sequenced

153 three new, rapidly evolving nuclear loci SF232, SF328 and SF412 (Tezuka et al., 2012), for a

154 total of 22, 23 and 25 additional DNA sequence fragments, respectively (the differences in the 
155 numbers of genes or individuals sequenced across loci were due to difficulties with the PCR

156 amplification). GenBank accession numbers, field and museum voucher codes, GPS coordinates,

157 and new data obtained for this study are provided in Table S1 in the supplementary material (see

158 supplementary Table S2 for PCR primers and molecular protocols). The purified fragments were

159 Sanger-sequenced in both directions. The cleaned forward and reverse strands were compared

160 before assembling consensus sequences using Geneious version 6.0 (Kearse et al., 2012).

161 Sequences were aligned using MAFFT version 7 (Katoh \& Standley, 2013) and corrected by eye

162 in Mesquite version 3.04 (Maddison \& Maddison, 2007).

163 In order to assess the genetic isolation and historical demography of Rheobates

164 populations, we evaluated the genetic structure for each locus, among locations and constructed a

165 median-joining haplotype network (Bandelt et al., 1999) implemented and visualized in the

166 software PopArt (Leigh \& Bryant, 2015).

168 Evolutionary genetic analyses

169 The best-fitting model of nucleotide substitution was selected for each gene alignment

170 with jModelTest version 2.1 (Darriba et al., 2012) using the corrected Akaike information

171 criterion (see the models selected in Table S3)

172 To determine the major lineages in the genus Rheobates, we performed a species tree

173 analysis using the software *BEAST (Heled \& Drummond, 2010). *BEAST simultaneously

174 estimates the gene trees for each locus together with a species tree, by implementing a

175 multispecies coalescent process. The input file for *BEAST was created with BEAUti v1.8

176 (Drummond et al., 2012). We constrained the root age by using the results of Santos et al. (2009)

177 as a secondary calibration and set the prior distribution on the age of the most recent common 
178 ancestor (MRCA) of dendrobatoid frogs as a normal distribution with a mean of 43.7 million

179 years ago (Ma) and standard deviation of 6.7 Ma. The nucleotide substitution model for each

180 gene was set as above and a lognormal relaxed clock. The prior for the uncorrelated lognormal

181 relaxed clock mean was with a gamma distribution with shape 0.004 , a scale of 1000 and an

182 offset of 0 . A coalescence tree prior was selected for modeling individuals of the same

183 population. Two independent analyses were run, each one for 100 million iterations with a burn-

184 in of 100000 generations.

We also inferred phylogenetic relationships using maximum likelihood (ML) analysis as

186 implemented in RAxML version 8.0.19 (Stamatakis, 2014). We used the standard GTRGAMMA

187 substitution model on concatenated data matrix for both the mitochondrial and nuclear loci. We

188 ran two RAxML analyses. First, we ran 1000 replicate searches to find the optimal ML tree.

189 Second, we performed bootstrapping using the autoMRE option, which automatically determines

190 a sufficient number of bootstrap replicates. The bootstrap support values are shown on the

191 inferred best ML tree (Fig S1 and S2), and all trees were rooted using the outgroup Allobates

192 (Table S1).

193 The posterior sample of trees from *BEAST was checked for convergence and effective

194 sample size (ESS > 200) of the estimated parameters using Tracer version 1.7 (Andrew Rambaut

195 et al., 2018). The resulting species tree inference was summarized with TreeAnnotator version

1961.8 (Drummond et al., 2012) as a majority 50\% consensus rule tree and visualized with FigTree

197 version 1.4.2 (A Rambaut, 2014) and with DensiTree version 2.0.1 (Bouckaert, 2010) using the

198 same posterior MCMC sample from the *BEAST analysis.

200 Ortíz et al., 2015) by using the Bayesian species delimitation program (BPP) (Rannala \& Yang, 
201 2013; Yang \& Rannala, 2010), which assumes the gene trees evolves within the constraint of the 202 species tree, and a Poisson Tree Process (PTP) model, which infers molecular clades based on an 203 estimated phylogeny (Zhang et al., 2013). Given that different species delimitation methods can

204 present variability among the results, it is important to test multiple approaches for a better

205 support of the number of species (Ortiz \& Francke, 2016; Toussaint et al., 2015).

For the BPP, we assumed three contrasting sets of prior distributions on demographic

207 parameters representing three types of histories: small population size with shallow divergence

208 among species, large population size with deep divergence, and large population size with

209 shallow divergences (Flouri et al., 2018; Yang, 2015). BPP uses the reversible-jump MCMC

210 algorithm which can exhibit mixing problems in some datasets (Rannala \& Yang, 2013; Yang \&

211 Rannala, 2010), and we therefore conducted the analyses three times, with different starting

212 seeds, to confirm consistency among runs. Although we only achieved ideal fine-tune adjustment

213 for the first scenario (small population size with shallow divergence), all runs consisted of 100

214000 samples with a burn-in period of 8000 steps.

215 For the PTP model, we used the RAxML topology to test for species delimitation, as

216 advocated for this method. We ran this analysis on the PTP webpage (Zhang et al., 2013)

217 adopting a burn-in period of $10 \%$ with thinning of 1 tree every 100 generations for 100000

218 generations, for a total of 900 posterior samples. To check for convergence, we did a visual

219 inspection of the likelihood plot of each delimitation as indicated by the program.

220 We used the MCMC coalescent simulator, IMa2 (Hey, 2011), which estimates migration

221 rates between the genetic lineages under an isolation-with-migration model (Nielsen \& Wakeley,

222 2001) to evaluate asymmetric migration rates between sister clades within Rheobates (see

223 Results). Runs were conducted with 100000 MCMC generations and a thinning of 100 with a 
224 burn-in of 10 percent. Following recommendations in the manual, we assumed theta $=10$, for the

225 parameter of maximum population size $(4 N \mu)$ and asymmetrical migrations $(\mathrm{m} 0 \rightarrow 1=\mathrm{m} 1 \rightarrow 0)$

226 (Liao et al., 2012) between pairs of locations.

\section{Landscape genetics}

We evaluated the potential abiotic drivers of genetic divergence by estimating the

230 relationship between geographic, environmental, least-cost path (LCP), and circuit distances

231 against genetic distances among all pairs of sampled individuals within the genus Rheobates, i.e.,

232 regardless of whether samples were currently assigned to $R$. palmatus or to $R$. pseudopalmatus

233 (Table S1). We estimated genetic distances between individuals with one concatenated dataset

234 for nuclear and one concatenated dataset for mitochondrial genes. The model selection and

235 genetic distances for each concatenated dataset were estimated in MEGA (Kumar et al., 2016), in

236 which TN93+ $+\mathrm{I}$ was the best model for mitochondrial genes and $\mathrm{HKY}+\mathrm{I}$ for the nuclear genes.

237 In order to spatially visualize the pairwise genetic distances within Rheobates we used the

238 software MAPI version 1.0.1 (Piry et al., 2016). MAPI implements a spatial network in which

239 samples are linked by ellipses and grids of hexagonal cells encompassing the study area. The

240 pairwise genetic distances attributed to ellipses are averaged and assigned to cells they intersect

241 following the principle that the larger the ellipse, the smaller its contribution to cells below it

242 (Piry et al., 2016). Cells with higher dissimilarity values indicate that geographically closer

243 individuals are more genetically different. The null distribution for the test of significance of

244 genetic structure, i.e., the spatial genetic discontinuities that are higher than expected by chance,

245 is generated through a randomization procedure. We used a beta of $0.25,10000$ permutations, 246 and an alpha set to 0.05 . 
248 pairwise localities using the software Geographical Distance Matrix Generator, version 1.2.3

249 (Ersts, 2012), which takes into account the Earth's curvature.

250 Environmental distances (to test for isolation by environment) were estimated by

251 extracting from each sampling site the 19 bioclimatic variables available in the WorldClim

252 database (http://www.bioclim.org) at 30 arcseconds resolution, using the function dist in the

253 RStudio (RStudio Team, 2020) statistical package version 3.6.0 (Wang, 2013). This function

254 estimates Euclidean pairwise distances in multidimensional space between rows on a

255 multivariate matrix, which indicate to how different the 19 bioclimatic variables are between

256 sampling sites, where smaller distances indicate more similar environments.

257 Circuit distances (to estimate isolation by resistance) were estimated by calculating a

258 resistance matrix as the inverse of a Rheobates species distribution model, assuming that areas

259 with low suitability have higher resistance to dispersal (Wang et al., 2008). The species

260 distribution model (SDM) was built using the MaxEnt algorithm (Phillips \& Dudík, 2008)

261 implemented in the Wallace package (Kass et al., 2018), again using the 19 bioclimatic variables

262 of WorldClim. Once the SDM was inferred, we obtained the resistance matrix by multiplying the

263 SDM by -1. Circuit distances were estimated using the software Circuitscape version 4.0.5

264 (McRae et al., 2013), which takes the resistance matrix and the geographic locations as input and

265 produces a matrix of resistance distances between sample pairs. This method allowed us to

266 evaluate the potential connectivity among samples by summarizing the costs of the resistance of

267 the landscape, taking into consideration multiple pathways, not just the 'least-costly'.

268 We also estimated LCPs to test for isolation by resistance. LCP distances account for the

269 movement of the animal as the 'least costly' or the more optimal pathway between pairs of 
270 samples (Wang et al., 2009). We calculated LCP using the 'gdistance' package for R and the

271 same resistance matrix used for the previous analysis.

272 We tested which of the previous spatial variables (geographic, environmental, circuit, or

273 LCPs) had the strongest association with genetic distances by using a multiple regression

274 approach. Because they were on different scales, we standardized the estimated distances by

275 subtracting the mean and dividing by the standard deviation. We then related the possible

276 associations of genetic distance with the four standardized matrices of abiotic variables using a

277 Multiple Matrix Regression with Randomization Analysis (MMRR) in R (Wang, 2013). Given

278 the nonindependence of the spatial variables, the MMRR analysis uses random permutations

279 (resampling without replacement) to generate null distributions. We conducted the MMRR

280 analysis with 10000 permutations, using the function provided by Wang (2013).

\section{Results}

283 The lineage from Santa María (east side of the Eastern Cordillera) was sister to the rest of

284 Rheobates, which was composed of four well-separated and largely allopatric lineages

285 identifiable by their geographic distribution. The Central Cordillera clade was separated from

286 those of the Eastern Cordillera which, in turn, can be divided into three clades. Two are found

287 north versus south of the Chicamocha Canyon, and the third is located further south (Fig. 1a).

288 The haplotype network inferred for each individual gene showed that the Santa María

289 lineage presented the most differences among haplotypes in both mitochondrial and nuclear

290 networks (Fig.1b).

291 The Bayesian multispecies coalescent analyses using *BEAST and the concatenated

292 RAxML inference showed almost identical topologies and identified five highly divergent 
293 lineages within the genus Rheobates. The major clades in which Rheobates were grouped

294 displayed high support (i.e., 100\% bootstrap support in the ML and posterior probability of 1 in

295 the Bayesian consensus tree). Figure 1c presents the consensus tree with posterior probabilities

296 on major nodes along with a graphical display of the topological variance within our posterior

297 sample of trees from the Bayesian analysis (Theys et al., 2019).

298 The BPP method of species delimitation showed that the five groups identified here have

299 a probability of $99.98 \%$ of representing distinct, unconfirmed candidate species, i.e., Santa

300 María, the Central Cordillera, plus the three clades distributed along the western flank of the

301 Eastern Cordillera that formed potentially allopatric replacements of each other along a north-

302 south transect. Based on both the multispecies coalescent and the concatenated phylogenetic

303 inference, PTP also recovered five unconfirmed candidate species, each supported by a posterior

304 probability of 1.

Historical demographic analyses of coalescent models of isolation with migration using

306 IMa2 revealed for most pairwise comparisons a migration rate $(\mathrm{Nm})$ indistinguishable from zero.

307 The highest of these low migration rate estimates was between Eastern South and Eastern

308 Central with $N m=0.178$ (95\% confidence interval of 0 to 0.716$)$ migrants per generation (Table

309 1). Low migration rates (<0.2 migrants per generation) most likely did not impede species

310 delimitation (Flouri et al., 2018).

311 The spatial visualization of the pairwise genetic distances made with MAPI (Fig. 2)

312 indicated that the largest genetic discontinuities per cell are in the northern part of the Magdalena

313 Valley and in the areas surrounding the locality of Santa María, in the south-eastern side of the

314 Eastern Cordillera. This region was also the only region with higher genetic discontinuities than

315 expected by chance (depicted with a black line in Fig. 2). In contrast, the areas with fewer 
316 genetic discontinuities were within the northern part of the Central Cordillera, and in the

317 northern and southern extremes of the Eastern Cordillera. A visual examination indicates that the

318 genetic discontinuities within Rheobates seem to match better with precipitation than

319 temperature (Fig. 2).

The MMRR analysis revealed that environmental distance, in other words, the degree of

321 climatic differentiation between sampling localities independently of the intervening areas that

322 separate localities, had a strong and significant regression coefficient $(\beta)$ in both nuclear and

323 mitochondrial loci (Nuclear genes: $\beta=3.091, P<0.003$; mitochondrial genes: $\beta=3.115, P<$

324 0.002). Geographic distance had a strong regression coefficient on the mitochondrial genes $(\beta=$

$3253.171, P<0.003)$, but was not significant for the nuclear genes $(\beta=1.211, P=0.228)$. Circuit

326 distance (Fig. 3) was significant for the nuclear genes $(\beta=-2.320, P<0.003)$, but was not

327 significant for the mitochondrial genes $(\beta=-0.793, P=0.428)$. LCP distance was not significant

328 for either group of genes (mitochondrial: $\beta=-0.410, P=0.678$; nuclear: $\beta=-0.555, P=0.574$ ).

330 Discussion

331 Rheobates is a montane genus of frog distributed across two of the main mountain ranges

332 of the Colombian Andes. Populations of Rheobates are separated by low, warm, and dry valleys

333 such as the Magdalena Valley and the Chicamocha Canyon, and in one case by high elevation

334 and cold peaks, namely the Eastern Cordillera ridge. In this study we explored how these

335 complex Neotropical landscapes may drive genetic divergence within the genus. We find

336 evidence that supports more lineages (i.e., unconfirmed candidate species) than were previously

337 expected. Moreover, we find evidence that individuals from sites with more similar 
338 environments are more genetically similar, which supports an isolation by environment scenario

339 in Rheobates.

340 In terms of the lineages within the genus, the species delimitation analyses, BPP and PTP,

341 supported with high confidence that Rheobates is composed by five reciprocally monophyletic

342 lineages that very likely correspond to distinct species, contrasting with the three species

343 suggested by Muñoz et al. (2015). In addition to the high genetic divergence (Fig. 1), these five

344 lineages displayed migration rate point estimates of $\mathrm{Nm}<0.006$ that were statistically

345 indistinguishable from zero, further supporting their status as unnamed species. Rheobates

346 appears to be very old. Muñoz et al. (2015) estimated the crown age of Rheobates at $21 \mathrm{Ma}$ and

347 estimated the ages of the younger three lineages of the Eastern Cordillera at around 7 to 9 Ma,

348 once again supporting the idea that all five lineages may be species. Gene trees of three out of

349 four nuclear loci (Fig. S2) and the mitochondrial genes (Fig. S1) recovered all five groups as

350 monophyletic, as well. Future studies based on other sources of data (e.g., male advertisement

351 calls, adult morphology, and larval morphology) are needed to test our hypothesis that these five

352 lineages found herein can be confirmed as candidate species (Padial et al., 2010) and then

353 formally described.

At first glance, geographic barriers stemming from the complex topography of the Andes

355 would seem to be the cause of the diversification within Rheobates. For example, the most

356 divergent lineage within Rheobates corresponds to the locality of Santa María, our only locality

357 on the eastern slope of the Eastern Cordillera (Fig 1), which may have diverged during the

358 Miocene (Muñoz-Ortíz et al., 2015). The second most divergent group corresponds to the Central

359 Cordillera, which is geographically separated from the remaining clades by the Magdalena

360 Valley. A similar pattern occurs among the three clades of the western slopes of the Eastern 
361 Cordillera. The lineage north of the Chicamocha Canyon is genetically well differentiated from

362 populations south of the Canyon (Fig.1). However, if geographic barriers were the main factor

363 promoting genetic divergence in Rheobates, we would have found support for isolation by

364 resistance. Instead, we found strong support only for isolation by environment (across nuclear

365 and mitochondrial loci). Thus, rather than the intervening areas between populations (geographic

366 barriers), the local environment promotes divergence independently of geographic distance or the

367 environmental 'resistance' between populations. Our depiction of spatial genetic discontinuities

368 supports this view (Fig. 2), showing that the genetic differentiation across the landscape is lower

369 when temperature and precipitation are more similar and higher when they are more different.

370 Interestingly, the genetic distances in both nuclear and mitochondrial DNA seem to be matching

371 better precipitation than temperature (Fig. 2).

Isolation by environment may result from local adaptation to particular environments or

373 selection against immigrants arriving at new sites (Nosil et al., 2005; Wang \& Bradburd, 2014).

374 Palm Rocket Frogs inhabit the edges of creeks and small ponds, and show a relatively high

375 tolerance towards considerable anthropic alterations of its environment (Cortés-Suárez, 2014;

376 Jérez \& Yara-Contreras, 2018). We are not aware of any studies looking explicitly at geographic

377 variation in the autecology of Rheobates, and are thus unsure of exactly which abiotic

378 differences among regions might be driving a pattern of isolation by environment. Based on two

379 studies of a few localities in the Eastern Cordillera, Rheobates apparently reproduces all year

380 long (Jérez \& Yara-Contreras, 2018; Lüddecke, 1999), making potential differences in

381 phenology between sites difficult to observed. Estimates of isolation by environment are derived

382 from SDMs based on abiotic factors, but these environmental variables could be spatially

383 correlated with local microclimatic conditions that could be limiting the gene flow via local 
384 adaptation. Microclimatic conditions shaping the distribution of organisms have previously been

385 shown for epiphytic bryophytes (León-Vargas et al., 2006), forest birds (Frey et al., 2016) and

386 frogs of the genus, Pristimantis (Pintanel et al., 2019), in which fine-scale metrics of temperature

387 and/or precipitation restrict the distribution of these organisms. Thus, finding a significant effect

388 of isolation by environment may not be that surprising, and should encourage us to investigate

389 other types of factors that may influence population isolation and divergence, beyond just

390 physical barriers, which also seem to be at play here.

391 We cannot reject outright the role of geographic barriers in promoting genetic

392 differentiation among populations, especially considering that topographic barriers and the

393 environmental barriers could be correlated. For example, the eastern slope of the Eastern

394 Cordillera is more humid than the western slope (Sklenár \& Lægaard, 2003). This is mostly due

395 to the rain shadow effect caused by the trade winds that come from the east (Mark \& Helmens,

396 2005). In this case the geographic variation in environment, i.e., higher humidity along the

397 eastern slope relative to the western slope, is due to a potential geographic barrier, i.e., the

398 Eastern Cordillera (but see Rodríguez-Muñoz et al., 2020). Assuming uniformitarianism, that the

399 same processes have been acting on Rheobates across its 35 million year history [the estimated

400 stem age reported by Muñoz-Ortiz et al. (2015)], a potential scenario would be that initial

401 divergences are driven by local adaptation to environmental variation while obvious landscape

402 features may arise secondarily, such as through the formation of valleys or mountain peaks that

403 secondarily act as barriers. Such a hypothesis would fit with what has been observed in

404 Neotropical birds, where the organism-environment interaction plays a more important role than

405 landscape features (Smith et al., 2014). 
Our multiple regression analysis revealed that isolation by distance was supported by the

407 mitochondrial loci but not by the nuclear loci, as with isolation by resistance, which was

408 supported by the nuclear loci but not the mitochondrial loci. Evolutionary processes (e.g., natural

409 selection and genetic drift) can act on mitochondrial and nuclear genomes differently.

410 Discordance between mitochondrial and nuclear gene regions have been frequently encountered

411 among animals including amphibians for almost two decades (Funk \& Omland, 2003; Toews \&

412 Brelsford, 2012). The discordances among loci in phylogeographic structure may result from

413 incomplete lineage sorting (Funk \& Omland, 2003), different effective population sizes (Zink \&

414 Barrowclough, 2008), natural selection (Irwin, 2002), life-history traits such as sex-biased

415 dispersal (Turmelle et al., 2011), coalescent variance (Lohse et al., 2010), selective sweep on a

416 specific area of the DNA (Bensch et al., 2006), or historic isolation and secondary contact (Petit

417 \& Excoffier, 2009). Future genomic level analyses and the use of microclimatic variables may

418 help to resolve the effect that each of these geographic features is having on the diversification of

419 this genus of frogs.

Historically, much attention has been focused on the effects of vicariance and geographic

421 distance as main factors promoting diversification in Neotropical mountains, and the case of the

422 Palm Rocket Frog would seem to be a prime example (Muñoz-Ortiz et al., 2015). In the present

423 study, however, we demonstrate that, even in Rheobates, environmental factors and ecological

424 adaptation, as revealed by the isolation by environment model, may be more important than

425 landscape features and vicariance (B. T. Smith et al., 2014) in promoting diversification. Our

426 results thus lend support to the growing idea in phylogeographic studies that the geographic

427 component of genetic diversity must be driven by the interaction between an organism and its

428 environment, an approach referred to as trait-based phylogeography (Paz et al., 2015). The 
429 challenge going forward will be to associate particular traits with reduced migration rates across

430 a heterogeneous environment, such as through landscape genomics (Bradburd et al., 2013). More

431 data on the habitats, life history, and the genomes of Neotropical frogs will revolutionize our

432 understanding of the origins of diversity.

\section{Acknowledgments}

435 We are very grateful to the following individuals: Elena Ritschard, Carlos José Pardo (Duke

436 University), Jonathan Syme (Flinders University) and Lisa Neyman (NOAA) for their comments

437 on earlier versions of the manuscript. We thank: Astrid Muñoz-Ortíz, Álvaro Andrés Velásquez,

438 María Alejandra Rueda, Philippe Genty, Martha E. Zapata, Luisa Dueñas, Camila Martínez,

439 Faidith Bracho (Universidad de Antioquia) and Santiago Herrera (University of Chicago) for

440 assistance. This research was submitted by G.G. to the Department of Biological Sciences of the

441 Universidad de los Andes in partial fulfilment of the requirements for the bachelor's degree.

443 Data availability statement

444 Tissue samples were obtained through museum donations from the Museo de Historia Natural C.

445 J. Marinkelle of the Universidad de los Andes. The supporting data generated here (i.e., DNA

446 sequence alignments, phylogenetic trees, geographic coordinates, SDM layers, and R script) will

447 be available on Dryad Digital Repository upon acceptance of this MS. 
448

\section{References}

Bandelt, H.-J., Forster, P., \& Röhl, A. (1999). Median-joining networks for inferring intraspecific phylogenies. Molecular Biology and Evolution, 16(1), 37-48.

Beebee, T. (2005). Conservation genetics of amphibians. Heredity, 95(6), 423-427.

Bensch, S., Irwin, D. E., Irwin, J. H., Kvist, L., \& Åkesson, S. (2006). Conflicting patterns of mitochondrial and nuclear DNA diversity in Phylloscopus warblers. Molecular Ecology, 15(1), 161-171.

Bernal, M. H., \& Lynch, J. D. (2008). Review and analysis of altitudinal distribution of the Andean anurans in Colombia. Zootaxa, 1826(1), 1-25.

Bouckaert, R. R. (2010). DensiTree: Making sense of sets of phylogenetic trees. Bioinformatics, 26(10), 1372-1373.

Bradburd, G. S., Ralph, P. L., \& Coop, G. M. (2013). Disentangling the effects of geographic and ecological isolation on genetic differentiation. Evolution, 67(11), 3258-3273.

Chaves, J. A., Weir, J. T., \& Smith, T. B. (2011). Diversification in Adelomyia hummingbirds follows Andean uplift: Hummingbird Diversification in the Andes. Molecular Ecology, $20(21), 4564-4576$.

Cortés-Suárez, J. E. (2014). Microhabitat use of Rheobates palmatus (Werner 1899)(Anura: Aromobatidae) in a riverside ecosystem of Villa De Leyva, Colombia. Herpetotropicos, 10(1-2), 5-7.

Darriba, D., Taboada, G. L., Doallo, R., \& Posada, D. (2012). jModelTest 2: More models, new heuristics and parallel computing. Nature Methods, 9(8), 772-772.

Drummond, A. J., Suchard, M. A., Xie, D., \& Rambaut, A. (2012). Bayesian phylogenetics with BEAUti and the BEAST 1.7. Molecular Biology and Evolution, 29(8), 1969-1973. 
Edwards, S., \& Bensch, S. (2009). Looking forwards or looking backwards in avian phylogeography? A comment on Zink and Barrowclough 2008. Molecular Ecology, Http://Biodiversityinformatics. Amnh.OOrg/Open_source/Gdmg. American Museum of Natural History.

Feder, M. E., \& Burggren, W. W. (1992). Environmental physiology of the amphibians. University of Chicago Press, Chicago, IL, USA.

Flouri, T., Jiao, X., Rannala, B., \& Yang, Z. (2018). Species Tree Inference with BPP Using Genomic Sequences and the Multispecies Coalescent. Molecular Biology and Evolution, 35(10), 2585-2593.

Folguera, A., \& Ramos, V. A. (2011). Repeated eastward shifts of arc magmatism in the Southern Andes: A revision to the long-term pattern of Andean uplift and magmatism. Journal of South American Earth Sciences, 32(4), 531-546.

Frey, S. J., Hadley, A. S., \& Betts, M. G. (2016). Microclimate predicts within-season distribution dynamics of montane forest birds. Diversity and Distributions, 22(9), 944-959.

Funk, D. J., \& Omland, K. E. (2003). Species-level paraphyly and polyphyly: Frequency, causes,

490 Ghalambor, C. K., Huey, R. B., Martin, P. R., Tewksbury, J. J., \& Wang, G. (2006). Are mountain passes higher in the tropics? Janzen's hypothesis revisited. Integrative and Comparative Biology, 46(1), 5-17. 
493 Graham, A. (2009). The Andes: A geological overview from a biological perspective. Annals of 494 the Missouri Botanical Garden, 96(3), 371-385.

495 Grant, P. R., \& Grant, B. R. (2011). How and why species multiply: The radiation of Darwin's 496 finches. Princeton University Press.

497 Grant, T., Frost, D. R., Caldwell, J. P., Gagliardo, R., Haddad, C. F., Kok, P. J., Means, D. B., 498 Noonan, B. P., Schargel, W. E., \& Wheeler, W. C. (2006). Phylogenetic systematics of 499 dart-poison frogs and their relatives (Amphibia: Athesphatanura: Dendrobatidae). Bulletin $500 \quad$ of the American Museum of Natural History, 2006(299), 1-262.

501 Grant, T., Rada, M., Anganoy-Criollo, M., Batista, A., Dias, P. H., Jeckel, A. M., Machado, D. J., \& Rueda-Almonacid, J. V. (2017). Phylogenetic systematics of dart-poison frogs and their relatives revisited (Anura: Dendrobatoidea). South American Journal of Herpetology,

Gutiérrez-Pinto, N., Cuervo, A. M., Miranda, J., Pérez-Emán, J. L., Brumfield, R. T., \& Cadena, C. D. (2012). Non-monophyly and deep genetic differentiation across low-elevation barriers in a Neotropical montane bird (Basileuterus tristriatus; Aves: Parulidae).

Hebert, P. D., Ratnasingham, S., \& De Waard, J. R. (2003). Barcoding animal life: Cytochrome c oxidase subunit 1 divergences among closely related species. Proceedings of the Royal Data. Molecular Biology and Evolution, 27(3), 570-580.

514 Hey, J. (2011). Documentation for IMA2. New Brunswick, NJ: Rutgers Uni. 
Hoorn, C., Wesselingh, F., Ter Steege, H., Bermudez, M., Mora, A., Sevink, J., Sanmartín, I., Sanchez-Meseguer, A., Anderson, C., \& Figueiredo, J. (2010). Amazonia through time: Andean uplift, climate change, landscape evolution, and biodiversity. Science, 330(6006), 927-931.

Horton, B. K., Parra, M., Saylor, J. E., Nie, J., Mora, A., Torres, V., Stockli, D. F., \& Strecker, M. R. (2010). Resolving uplift of the northern Andes using detrital zircon age signatures. GSA Today, 20(7), 4-10.

Irwin, D. E. (2002). Phylogeographic breaks without geographic barriers to gene flow. Evolution, 56(12), 2383-2394.

Jaramillo, C., Rueda, M. J., \& Mora, G. (2006). Cenozoic plant diversity in the Neotropics. Science, 311(5769), 1893-1896.

Jérez, A., \& Yara-Contreras, C. (2018). Rheobates palmatus (Werner, 1899). Catálogo de Anfibios y Reptiles de Colombia, 4, 68-78.

Kass, J. M., Vilela, B., Aiello-Lammens, M. E., Muscarella, R., Merow, C., \& Anderson, R. P. (2018). Wallace: A flexible platform for reproducible modeling of species niches and distributions built for community expansion. Methods in Ecology and Evolution, 9(4),

532 Katoh, K., \& Standley, D. M. (2013). MAFFT multiple sequence alignment software version 7: Improvements in performance and usability. Molecular Biology and Evolution, 30(4), 772-

535 Kearse, M., Sturrock, S., \& Meintjes, P. (2012). The Geneious 6.0. 3 read mapper. Biomatters Ltd.: Auckland, New Zealand. 
Kumar, S., Stecher, G., \& Tamura, K. (2016). MEGA7: Molecular evolutionary genetics analysis version 7.0 for bigger datasets. Molecular Biology and Evolution, 33(7), 1870-1874.

Leier, A., McQuarrie, N., Garzione, C., \& Eiler, J. (2013). Stable isotope evidence for multiple pulses of rapid surface uplift in the Central Andes, Bolivia. Earth and Planetary Science

Leigh, J. W., \& Bryant, D. (2015). PopART: Full-feature software for haplotype network construction. Methods in Ecology and Evolution, 6(9), 1110-1116.

León-Vargas, Y., Engwald, S., \& Proctor, M. C. (2006). Microclimate, light adaptation and desiccation tolerance of epiphytic bryophytes in two Venezuelan cloud forests. Journal of

Liao, P.-C., Tsai, C.-C., Chou, C.-H., \& Chiang, Y.-C. (2012). Introgression between cultivars

Lohse, K., Sharanowski, B., \& Stone, G. N. (2010). Quantifying the Pleistocene history of the oak gall parasitoid Cecidostiba fungosa using twenty intron loci. Evolution: International

Lüddecke, H. (1999). Behavioral aspects of the reproductive biology of the Andean frog Colostethus palmatus (Amphibia: Dendrobatidae). Revista de La Academia Colombiana de Ciencias Exactas, Físicas y Naturales, 23(suplemento), 303-316. evolution but not speciation rates in Amazonian birds. Ecology Letters, 22(4), 624-633.

Maddison, W., \& Maddison, D. (2007). Mesquite: A modular system for evolutionary analysis. 2011. See Http://Mesquiteproject. Org. 
560

561

562

563

564

565

566

567

568

569

Mark, B. G., \& Helmens, K. F. (2005). Reconstruction of glacier equilibrium-line altitudes for the Last Glacial Maximum on the High Plain of Bogotá, Eastern Cordillera, Colombia: Climatic and topographic implications. Journal of Quaternary Science: Published for the Quaternary Research Association, 20(7-8), 789-800.

McRae, B. H. (2006). Isolation by resistance. Evolution, 60(8), 1551-1561.

McRae, B., Shah, V., \& Mohapatra, T. (2013). Circuitscape 4 user guide. The nature conservancy.

Mescua, J. F., Giambiagi, L. B., \& Ramos, V. A. (2013). Late Cretaceous uplift in the Malargüe fold-and-thrust belt $\left(35^{\circ} \mathrm{S}\right)$, southern Central Andes of Argentina and Chile. Andean Geology, 40(1), 102-116.

Muir, A. P., Biek, R., Thomas, R., \& Mable, B. K. (2014). Local adaptation with high gene flow: Temperature parameters drive adaptation to altitude in the common frog (Rana temporaria). Molecular Ecology, 23(3), 561-574.

Muñoz-Ortíz, A., Velásquez-Álvarez, Á. A., Guarnizo, C. E., \& Crawford, A. J. (2015). Of peaks and valleys: Testing the roles of orogeny and habitat heterogeneity in driving allopatry in mid-elevation frogs (Aromobatidae: Rheobates) of the northern Andes. Journal of Biogeography, 42(1), 193-205.

Nielsen, R., \& Wakeley, J. (2001). Distinguishing migration from isolation: A Markov chain Monte Carlo approach. Genetics, 158(2), 885-896.

Nosil, P., Vines, T. H., \& Funk, D. J. (2005). Reproductive isolation caused by natural selection against immigrants from divergent habitats. Evolution, 59(4), 705-719. 
581 Ortiz, D., \& Francke, O. F. (2016). Two DNA barcodes and morphology for multi-method 582 species delimitation in Bonnetina tarantulas (Araneae: Theraphosidae). Molecular Phylogenetics and Evolution, 101, 176-193.

Ovalle-Pacheco, A., Camacho-Rozo, C., \& Arroyo, S. (2019). Amphibians from Serrania de Las Quinchas, in the mid-Magdalena river valley, Colombia. Check List, 15, 387.

Padial, J. M., Miralles, A., De la Riva, I., \& Vences, M. (2010). The integrative future of taxonomy. Frontiers in Zoology, 7(1), 1-14.

Paz, A., Ibáñez, R., Lips, K. R., \& Crawford, A. J. (2015). Testing the role of ecology and life history in structuring genetic variation across a landscape: A trait-based phylogeographic Evolution, 24(7), 386-393.

Phillips, S. J., \& Dudík, M. (2008). Modeling of species distributions with Maxent: New extensions and a comprehensive evaluation. Ecography, 31(2), 161-175.

Pintanel, P., Tejedo, M., Ron, S. R., Llorente, G. A., \& Merino-Viteri, A. (2019). Elevational and microclimatic drivers of thermal tolerance in Andean Pristimantis frogs. Journal of Biogeography, 46(8), 1664-1675.

601 Rambaut, A. (2014). FigTree 1.4. 2 software. Institute of Evolutionary Biology, Univ. Edinburgh.

602 Rambaut, Andrew, Drummond, A. J., Xie, D., Baele, G., \& Suchard, M. A. (2018). Posterior summarization in Bayesian phylogenetics using Tracer 1.7. Systematic Biology, 67(5), 901. 
604 Rannala, B., \& Yang, Z. (2013). Improved reversible jump algorithms for Bayesian species

605 delimitation. Genetics, 194(1), 245-253.

606 Rivero, J. A., \& Serna, M. A. (1995). Nuevos Colostethus (Amphibia, Dendrobatidae) del

607 Departamento de Antioquia, Colombia, con la descripción del renacuajo de Colostethus

608 fraterdanieli. Revista de Ecología Latinoamericana, 2(1-3), 45-58.

609 Rodríguez-Muñoz, E., Montes, C., \& Crawford, A. J. (2020). Synthesis of geological and comparative phylogeographic data point to climate, not mountain uplift, as driver of divergence across the Eastern Andean Cordillera. BioRxiv, 2020.01.14.906982. https://doi.org/10.1101/2020.01.14.906982

RStudio Team. (2020). RStudio: Integrated development for R. RStudio Inc.(2015). (FCT), Azores.

Santos, J. C., Coloma, L. A., Summers, K., Caldwell, J. P., Ree, R., \& Cannatella, D. C. (2009).

619 Schluter, D. (2000). The ecology of adaptive radiation. OUP Oxford.

620 Sklenár̆, P., \& Lægaard, S. (2003). Rain-shadow in the high Andes of Ecuador evidenced by paramo vegetation. Arctic, Antarctic, and Alpine Research, 35(1), 8-17. Perez-Eman, J., Burney, C. W., Xie, X., \& Harvey, M. G. (2014). The drivers of tropical speciation. Nature, 515(7527), 406-409. 
625 Smith, M. A., \& Green, D. M. (2005). Dispersal and the metapopulation paradigm in amphibian ecology and conservation: Are all amphibian populations metapopulations? Ecography, large phylogenies. Bioinformatics, 30(9), 1312-1313.

Tezuka, A., Matsushima, N., Nemoto, Y., Akashi, H. D., Kawata, M., \& Makino, T. (2012). organisms. PloS One, 7(2).

Theys, K., Lemey, P., Vandamme, A.-M., \& Baele, G. (2019). Advances in Visualization Tools for Phylogenomic and Phylodynamic Studies of Viral Diseases. Frontiers in Public Health, 7.

Toews, D. P., \& Brelsford, A. (2012). The biogeography of mitochondrial and nuclear discordance in animals. Molecular Ecology, 21(16), 3907-3930.

Toussaint, E. F., Morinière, J., Müller, C. J., Kunte, K., Turlin, B., Hausmann, A., \& Balke, M. (2015). Comparative molecular species delimitation in the charismatic Nawab butterflies (Nymphalidae, Charaxinae, Polyura). Molecular Phylogenetics and Evolution, 91, 194-

Turmelle, A. S., Kunz, T. H., \& Sorenson, M. D. (2011). A tale of two genomes: Contrasting patterns of phylogeographic structure in a widely distributed bat. Molecular Ecology,

645 Wang, I. J. (2013). Examining the full effects of landscape heterogeneity on spatial genetic variation: A multiple matrix regression approach for quantifying geographic and ecological isolation. Evolution, 67(12), 3403-3411. 
648 Wang, I. J., \& Bradburd, G. S. (2014). Isolation by environment. Molecular Ecology, 23(23), $5649-5662$.

650 Wang, I. J., Glor, R. E., \& Losos, J. B. (2013). Quantifying the roles of ecology and geography in spatial genetic divergence. Ecology Letters, 16(2), 175-182.

652 Wang, I. J., Savage, W. K., \& Bradley Shaffer, H. (2009). Landscape genetics and least-cost path analysis reveal unexpected dispersal routes in the California tiger salamander (Ambystoma

Wang, Y.-H., Yang, K.-C., Bridgman, C. L., \& Lin, L.-K. (2008). Habitat suitability modelling to correlate gene flow with landscape connectivity. Landscape Ecology, 23(8), 989-1000.

657 Weir, J. T., \& Price, M. (2011). Andean uplift promotes lowland speciation through vicariance and dispersal in Dendrocincla woodcreepers: Vicariance and dispersal in Dendrocincla woodcreepers. Molecular Ecology, 20(21), 4550-4563.

Werner, F. (1899). Ueber reptilien und Batrachier aus columbien und trinidad. Verhandlungen Des Zoologisch-Botanischen Vereins in Wien, 49, 470-484.

662 Wright, S. (1943). Isolation by distance. Genetics, 28(2), 114.

663 Yang, Z. (2015). The BPP program for species tree estimation and species delimitation. Current Zoology, 61(5), 854-865.

665 Yang, Z., \& Rannala, B. (2010). Bayesian species delimitation using multilocus sequence data. Proceedings of the National Academy of Sciences, 107(20), 9264-9269.

667 Zhang, J., Kapli, P., Pavlidis, P., \& Stamatakis, A. (2013). A general species delimitation method 668 with applications to phylogenetic placements. Bioinformatics, 29(22), 2869-2876. phylogeography. Molecular Ecology, 17(9), 2107-2121. 
Figure 1. a) Map of the Eastern and Central Andean cordilleras of Colombia indicating the sampling localities. The yellow line represents the Chicamocha Canyon. The red star shows the location of Bogotá, the capital of Colombia. The colors of the sampling localities match the haplotypes in Fig. 1b and geographic clades in Fig. 1c. b) Haplotype networks of Rheobates spp. of the concatenated mitochondrial sequences (COI and 16S) on the left side and concatenated nuclear sequences on the right side (POMC, SF232, SF328 and SF412). Circle area is proportional to the number of individuals in possession of the particular haplotype sequence. Each red number on a branch represents the mutational steps between haplotypes. Branches without red numbers have one mutational step between the haplotypes. c) Densitree with five major clades named according to geographic range and Bayesian consensus tree with posterior probabilities for the major clade. 
(a)

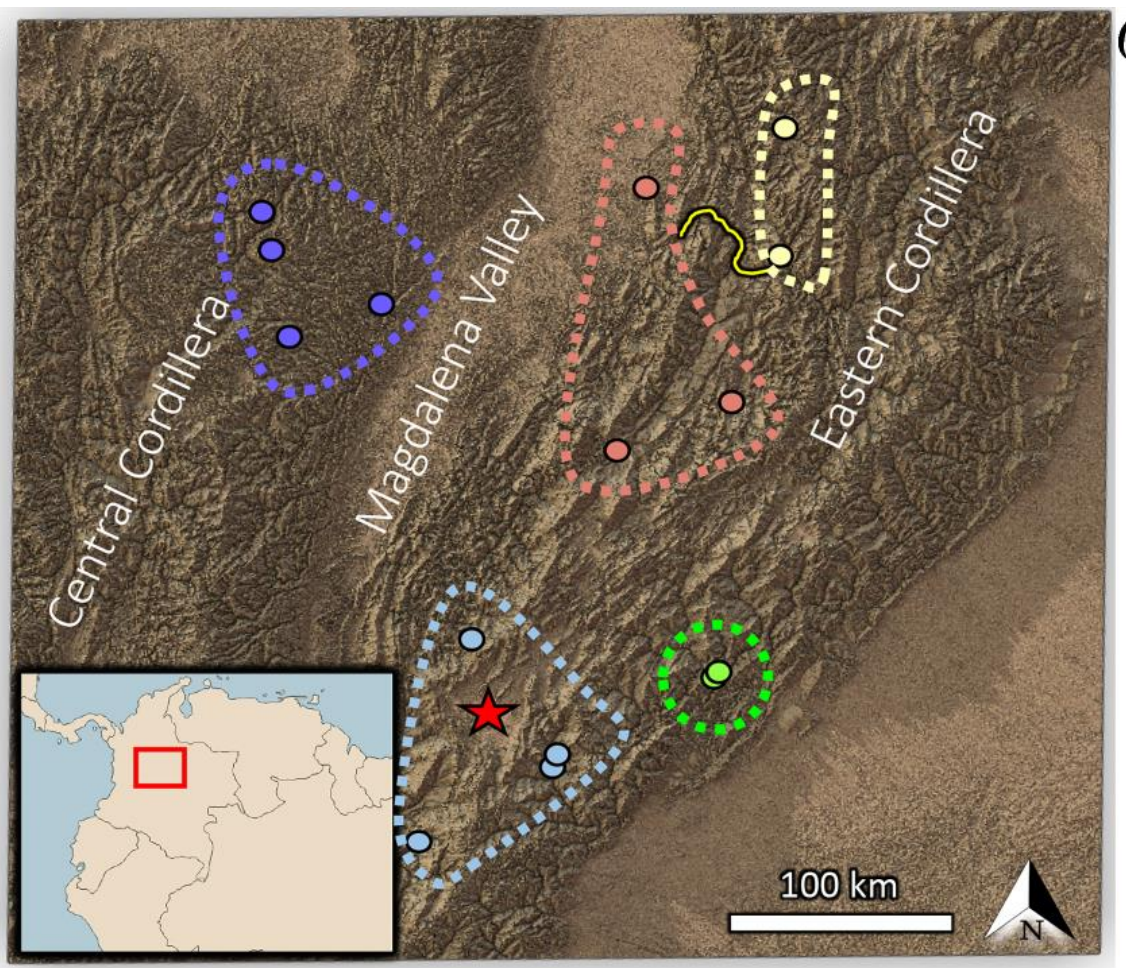

(b)

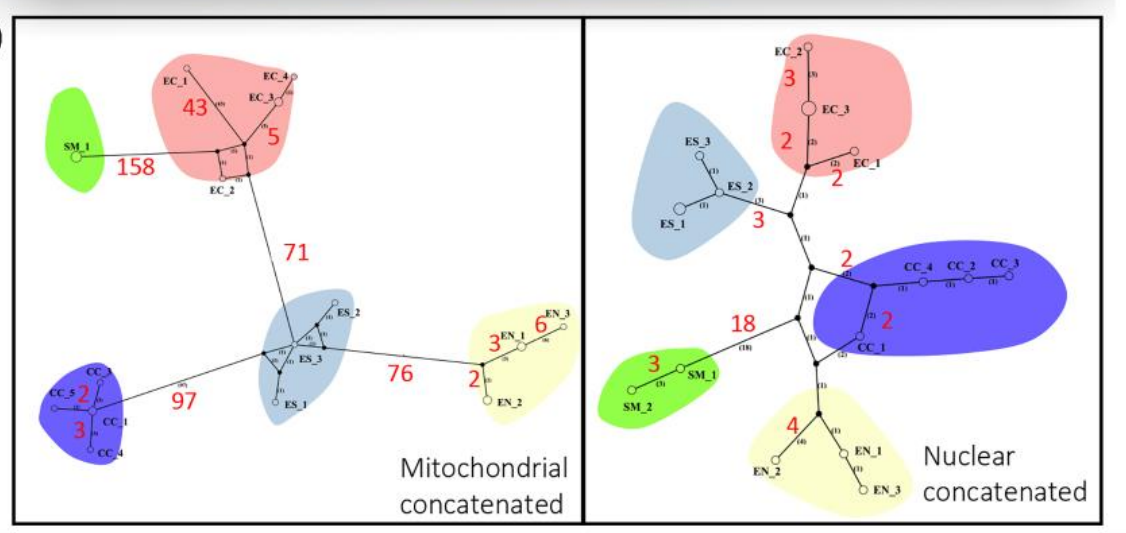

(c)

ANDES-A 1487

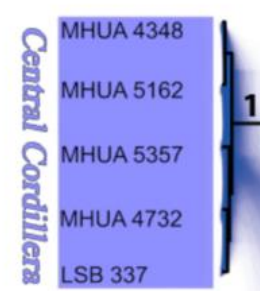

MAR 2175

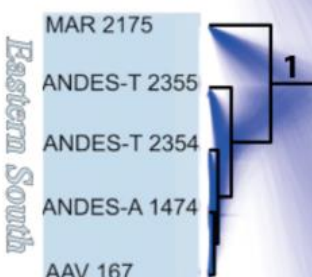

AAV 167

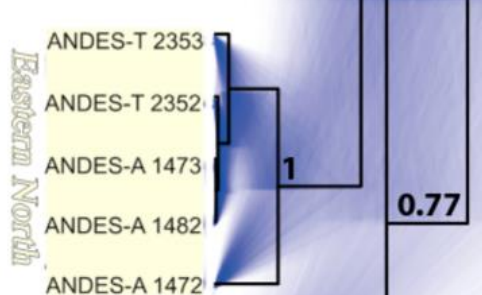

ANDES-A 1472

(t) ANDES-A 1481

ANDES-T 2350

ANDES-A 1478

AND

ANDES-A 1476
1

1

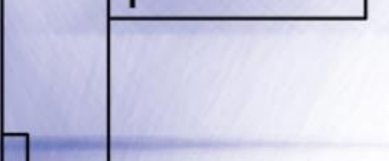


Figure 2. Left: MAPI spatial structure output. Polygons with black contours near Santa María correspond to areas with significantly higher inter-individual dissimilarity than expected by chance. The levels of genetic dissimilarity (MAPI values) range from red (lower genetic dissimilarity) to blue (higher genetic dissimilarity). Genetic distances were estimated with the mitochondrial and nuclear dataset. The circles on the figure indicates the sampling localities depicted in Fig. 1. Center: The current annual temperature (WorldClim dataset) of the same region as depicted on the left. Right: The precipitation (WordClim dataset) of the same region as depicted on the left.
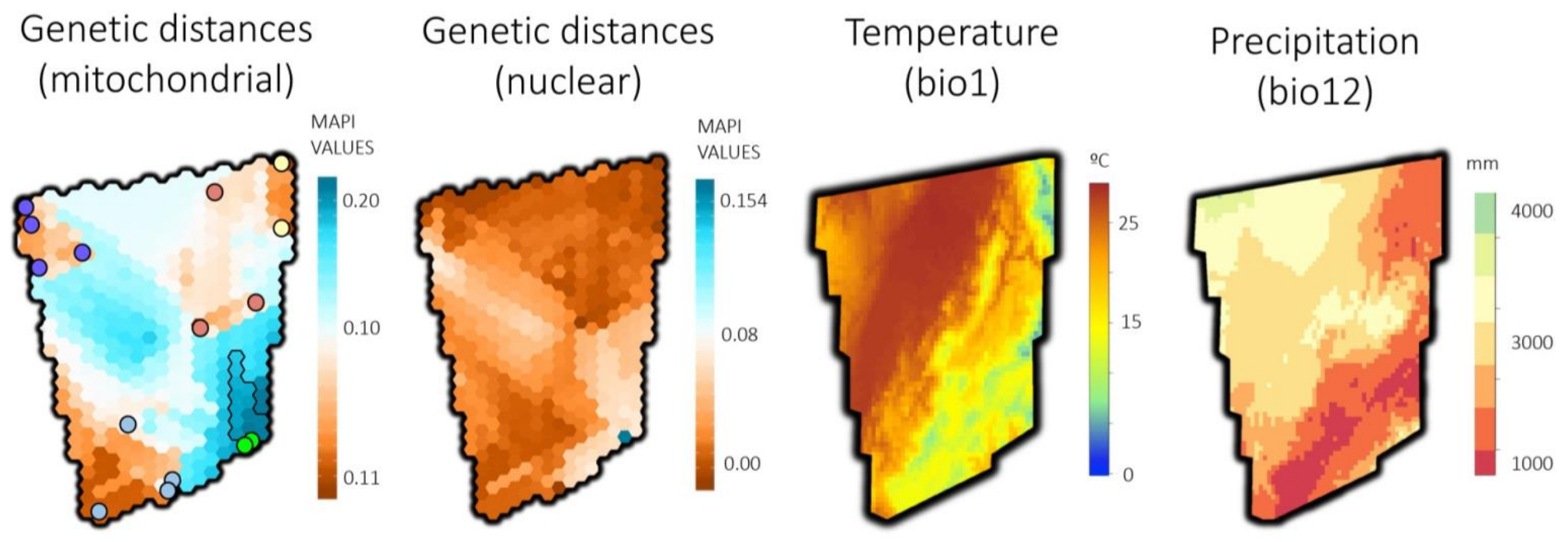
Table 1. We estimated the effective number of migrants $(\mathrm{Nem})$ between sister clades within

Rheobates. $95 \%$ credible intervals (CI) were calculated from posterior distribution of parameter estimates fitted to an isolation-with-migration model of historical demography in the Bayesian MCMC software, IMa2. Asymmetric migration rate estimates are shown here for migration from population 1 to 2 and from 2 to 1 .

\begin{tabular}{lll}
\hline \multirow{2}{*}{ Population pairs } & \multicolumn{2}{c}{ Migration (95\% CI) } \\
\cline { 2 - 3 } & $1->2$ & 2 -> 1 \\
\hline Santa María-Central Cordillera & $0.078(0.001-0.278)$ & $0.000(0-0.131)$ \\
Central Cordillera-Eastern Central & $0.024(0-0.500)$ & $0.000(0-0.292)$ \\
Eastern Central-Eastern South & $0.000(0-0.336)$ & $0.178(0-0.716)$ \\
Eastern Central-Eastern North & $0.087(0-0.647)$ & $0.000(0-0.774)$ \\
Eastern North-Eastern South & $0.000(0-0.578)$ & $0.000(0-0.601)$ \\
\hline
\end{tabular}

